\title{
BEAT CLASSIFICATION USING HYBRID WAVELET TRANSFORM BASED FEATURES AND SUPERVISED LEARNING APPROACH
}

\author{
M.Sasireka, Dr.A.Senthilkumar \\ Assistant Professor(Selection Grade), Department of EIE, Kongu Engineering College, Erode- \\ 638052.Tamilnadu.sasirek_28@yahoo.com \\ Professor and Head, Department of EEE, Dr. Mahalingam College of Engineering \& \\ Technology \\ Pollachi-642003.Tamilnadu.ask_rect@yahoo.com
}

\begin{abstract}
This paper describes an automatic heartbeat recognition based on QRS detection, feature extraction and classification. In this paper five different type of ECG beats of MIT BIH arrhythmia database are automatically classified. The proposed method involves QRS complex detection based on the differences and approximation derivation, inversion and threshold method. The computation of combined Discrete Wavelet Transform (DWT) and Dual Tree Complex Wavelet Transform (DTCWT) of hybrid features coefficients are obtained from the QRS segmented beat from ECG signal which are then used as a feature vector. Then the feature vectors are given to Extreme Learning Machine (ELM) and k- Nearest Neighbor $(\mathrm{kNN})$ classifier for automatic classification of heartbeat. The performance of the proposed system is measured by sensitivity, specificity and accuracy measures.
\end{abstract}

\section{INTRODUCTION}

The Electrocardiogram is the electrical activity of the heart which is very important to diagnose the heart disease. The early detection and treatment of ECG signal prevent the patient life from heart diseases and permanent damage from the tissues of the heart (Yuksel Ozbay \& Gulay Tezel, 2010) [1]. It is a very important tool to analyze the abnormality in ECG shape. Arrhythmia is a term used to define the ECG shape in abnormality condition. It is a common term in heart beat diagnosis and it differs from normal sinus rhythm (Tsipouras \& Fotiadis, 2004) [2].

There are several types of arrhythmia and each type is defined with pattern and is used to identify and classify its type. The arrhythmia comprises of two major types; the first type is formed by single irregular heartbeat and is called as morphological arrhythmia. The second type is formed by a set of irregular heartbeat and is called as rhythmic arrhythmias (Eduardo et al., 2015) [3]. The detection and classification of arrhythmias are more important in clinical cardiology in the time of real time application. This is obtained through extracted waves from ECG signal (Tsipouras et al., 2005) [4].

The main drawback in heart disease diagnosis is that the normal ECG signal is differ for each person and the symptoms of the heart disease abnormality is not shown all the time in ECG signal record. Due to this several hours is extended for successful diagnosis in manual investigation of ECG records. The manual system is a large time consuming as well as having a possibility of missing vital information (Yakup Kutlu \& Damla Kuntalp, 2011 and Berat Dogan \& Mehmet, 2012) $[5,6]$

To alleviate this problem, Computer-based automatic arrhythmia detection and classification system is needed in clinical. Computer based development methods used both signal processing and pattern recognition methods. There are two groups in pattern recognition system based on their learning procedures are supervised learning and unsupervised learning system. In supervised learning system training and testing are two types of modes used. A supervised learning system analyze the output based on training data, a training data is formed for all ECG signal in given database based on two classes. However, unsupervised learning system does not need any training data. It forms cluster from a given dataset based on similarity distance measure it gives the output.

In this paper a supervised learning algorithm is used for detection and classification of ECG signal record. The proposed system for arrhythmia classification from ECG signals in four steps as follows: (1) ECG signal preprocessing; (2) heartbeat segmentation; (3) feature extraction; and (4) identification/classification. In first phase Band pass filter is used to remove the unwanted signal in the ECG record. Segmentation of $Q, R, S$ waveform is described in second phase. Hybrid feature is used for the extraction of $P, Q, R, S$ waveform features and it is used as a input to the classification for identification of ECG signal. The final phase if the classification of ECG signals into normal or abnormal ECG signal based on unsupervised classifier system. The ECG signals in the MIT BIH database are used for accomplishing the proposed framework.

The aim of the paper is focused on classify the largest heartbeat cases in the MIT-BIH arrhythmia database such as (i) left bundle branch block (LBBB) (ii) right bundle branch block (RBBB) (iii) NORM (normal heartbeats).

The organization of the paper is as follows. In Section 2, describes about the literature survey of ECG signal analyses. The proposed method for classification of heartbeat cases is introduced in Section 3. Section 4 presents the MIT-BIH arrhythmia database which can be used to evaluate the effectiveness of the proposed method. In Section 5 , discussion and performance comparison are depicted. Finally, Section 6 presents conclusions.

\section{LITERATURE SURVEY}

The analysis of ECG signal for classifying the heartbeat cases has received considerable attention. (Koski, 1996) [7] proposed the Hidden Markov models for detection and classifying the heartbeats. (Elif Derya, 2009) [8] 
proposed adaptive neuro-fuzzy inference method for the classification of ECG signal. In this system Lyupanov exponent systems are used to extract the features of all ECG record. The system is classified by ANFIS and the training was done by the combination of back propagation gradient descent method and the least squares method.

(Ataollah et al., 2010) [9] proposed a system in the combination of preprocessing, feature extraction and classification. Stationary Wavelet transform (SWT) is used for removal of noise in the ECG signal. The combination of morphological based feature and timing interval based feature was proposed for extraction PQRS waveform amplitude. Finally four types of supervised classifier was used for classification of ECG signal are Multi-Layer Perceptron Neural Networks (MLPNN), Support Vector Machines (SVM), Radial Basis Function (RBF) and Probabilistic Neural Networks (PNN). (Homaeinezhad et al., 2012) [10] proposed a combination of SVM-kNN classifier and RBF-SVM classifier for detection and classification of ECG arrhythmia. (Mohit et al., 2017) [11] proposed Least Squares Support Vector Machine (LS-SVM) for the classification of coronary artery disease in ECG signal.

(Roshan et al., 2013) [12] proposed ECG beat classification method based on the combination of QRS detection, feature extraction, feature reduction and classification. QRS complex waveform is detected using Pan Tompkins algorithm. Discrete Wavelet Transform (DWT) is used to extract the features from QRS complex waveform and the obtained features are reduced using Principal Component Analysis (PCA-DWT), Linear Discriminant Analysis (LDA-DWT) and Independent Component Analysis (ICA-DWT). Finally reduced features are given to three types of classifier for ECG beat classifications are Support Vector Machine (SVM), Neural Network (NN) and Probabilistic Neural Network (PNN).

(Mehta \& Lingayat, 2008) [13] proposed a system for detection of ECG waveform consists of noise removal, Extraction of QRS wave form and classification. They used FIR notch filter for the removal of power line interference and base line wander. After that extraction of QRS waveform from denoised ECG signal and finally LIBSVM was used to classify the ECG signal.

(Yu \& Chen, 2007) [14] classified six types of arrhythmias based on RR intervals as well three types of statistical and detection of ECG using PNN. (Khazaee \& Ebrahimzadeh, 2010) [15] classified five types of arrhythmias based on feature extraction and classification where Non-parametric power spectral density (PSD) estimation methods are used to extract spectral features from ECG signal and hybrid optimized approach of hybrid genetic algorithm-support vector machines (SVMGA) was employed to classify the ECG signal.

\section{METHODOLOGY}

The overview of the proposed system for ECG signal classification has been shown in Fig.1. The ECG signals for training and testing dataset are taken from MIT-BIH database. The proposed ECG signal system is divided into three groups are preprocessing, feature extraction and classification. In training stage, all signals from MIT-BIH database are processed by preprocessing and feature extraction and formed feature vector. In testing stage the input signal from MIT$\mathrm{BIH}$ database is processed by first two groups and obtained features from feature extraction group is matched with feature vector from testing stage. The matching process is taken by supervised learning approach in classification stage and provides the result as ECG normal or ECG abnormal.

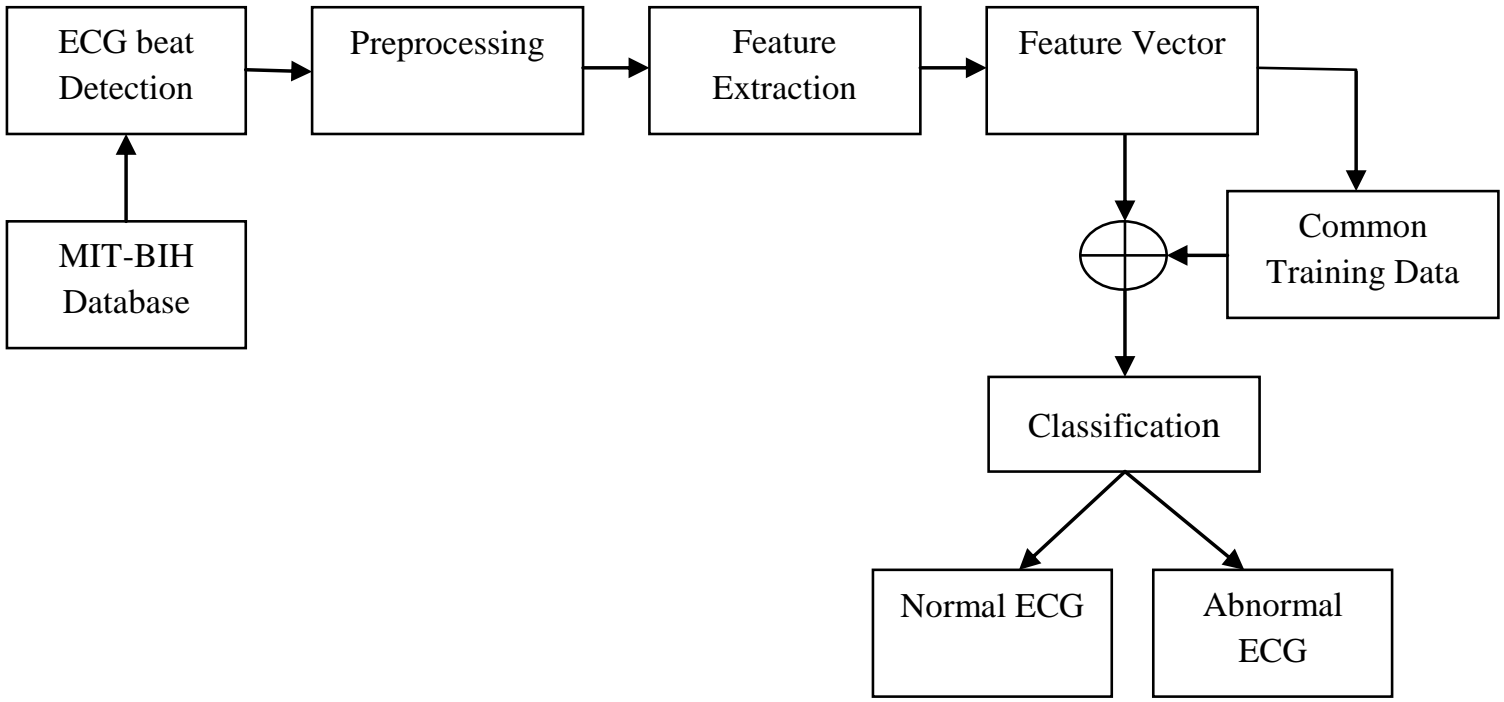

Figure 1: The Proposed ECG Signal Classification Steps

\subsection{Preprocessing of ECG signal}

ECG signal picked from MIT-BIH database is often contaminated by disturbances such as power line interference and baseline wander. This interference is occurred through changes in the electrode-to-skin polarization voltages. The baseline wander noise contains low frequency components while power line interference contains high frequency components (Yakup Kutlu \& Damla Kuntalp, 2011) [6]. The band pass filter is used to remove the baseline wander 
and power line interference in ECG signal. The ECG signal is filtered with band pass filter for removing EMG artifacts from muscles; motion artifacts from the subject and electrodes, baseline wander and power line interference.

\subsection{Detection of QRS Peak}

The QRS complex detection in an ECG signal is an important factor to diagnose the cardiac disease. QRS feature is most significant feature compared to any ECG feature because it provides the information of the heart rate computation. The QRS complex is based on the (i) Differences \& Approximation Derivatives, (ii) Inversion and (iii) Thresholding.

First calculate the difference between the adjacent elements of the ECG signal. If $X$ is a $1 D$ vector of an ECG signal then differences and approximation derivative of the filtered ECG signal is implemented as:

$$
Y 1=[X(2)-X(1) X(3)-X(2) \ldots . X(n)-X(n-1)]
$$

Once again differences and approximation derivation is calculated for obtained $\mathrm{Y} 1$ signal

$$
Y 2=[Y 1(2)-Y(1) Y 1(3)-Y 1(2) \ldots Y 1(n)-Y 1(n-1)]
$$

The output of $Y 1$ and $Y 2$ is cumulated for the detection of R-peak and is given as follows:

$$
Y=1.3 * Y 1(1: f s * 2)+1.1 *(1: f s * 2)
$$

Where $f s$ indicates the sampling frequency

The s-wave peak is detected by finding local minimum value on the inverted $\mathrm{Y}$ signal.

The detection of a Q-wave peak is achieved by comparing the threshold on minimum peak value on $Y$ signal. The thresholding scheme for searching the q-wave peak is formulated as:

$$
\begin{gathered}
\text { if } Y(m)>T H V \& Y(m)<T H V \rightarrow Q R S \text { complex } \\
\text { otherwise } \rightarrow \text { None }
\end{gathered}
$$

Where THV is the threshold herein the threshold are $0.2 \mathrm{mv}$ and $0.5 \mathrm{mv}$ and $\mathrm{m}$ is the minimum amplitude value in Y signal.

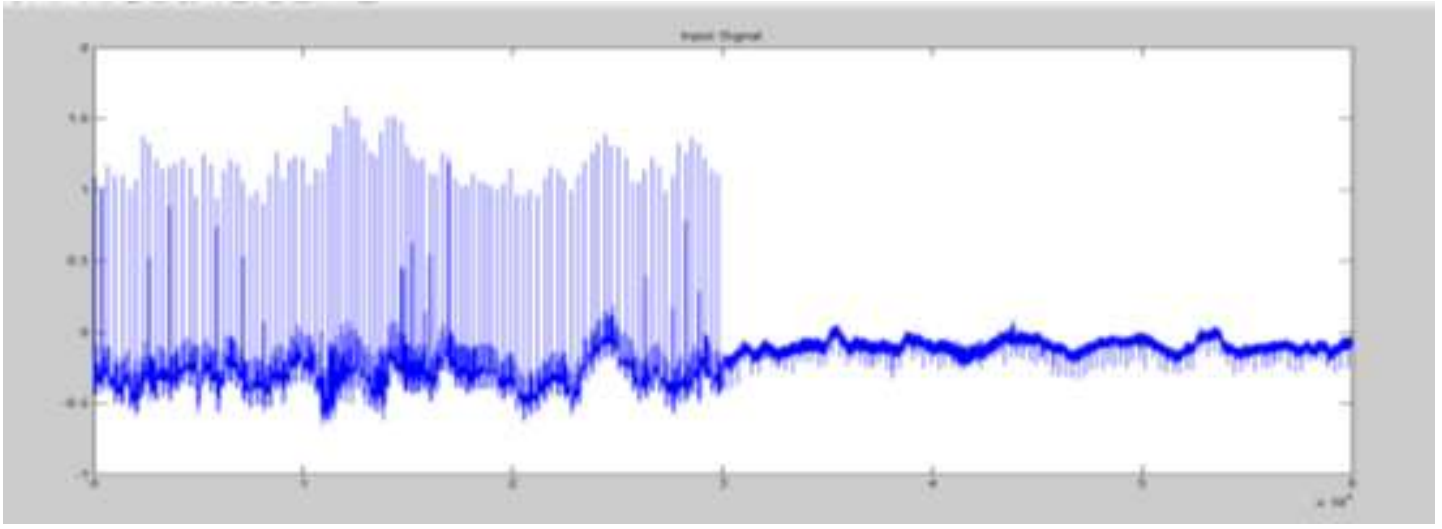

Figure 2: Input signal

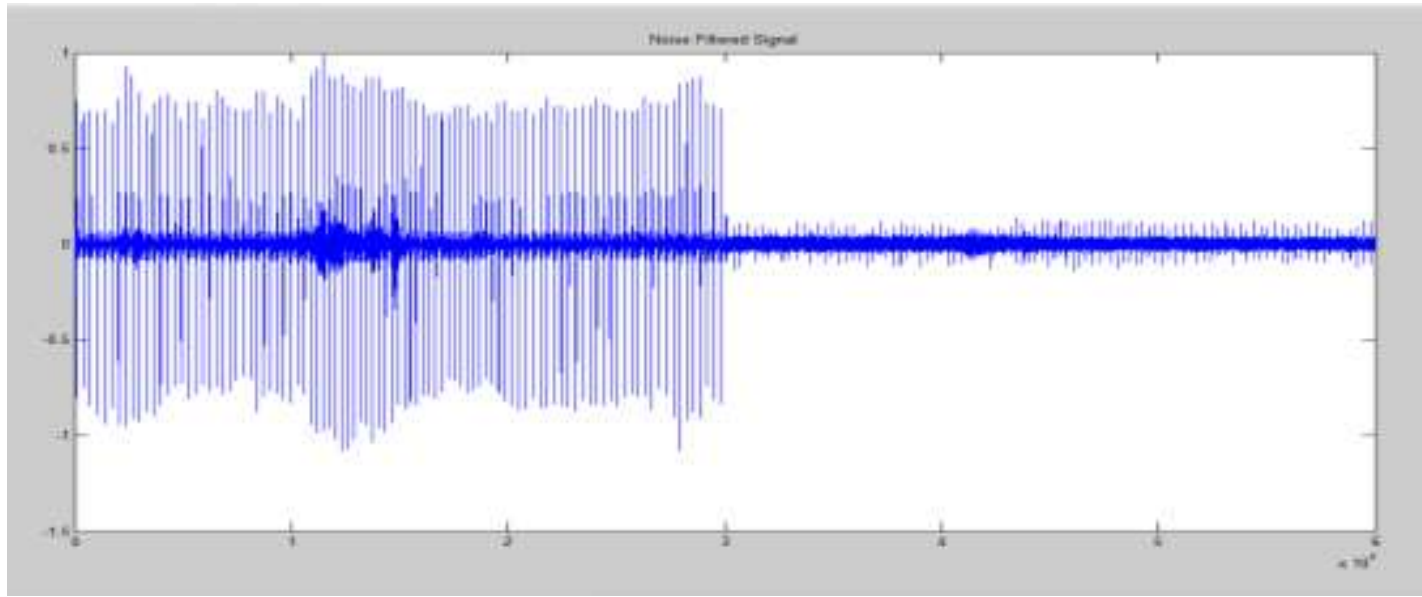

Figure 3: Filtered Output 
The ECG signal taken from the record 100 of MIT BIH database are shown in fig. 2. The filtered output done by band pass filter is shown in fig.3. The QRS complex of the filtered ECG signal is shown in fig.4.

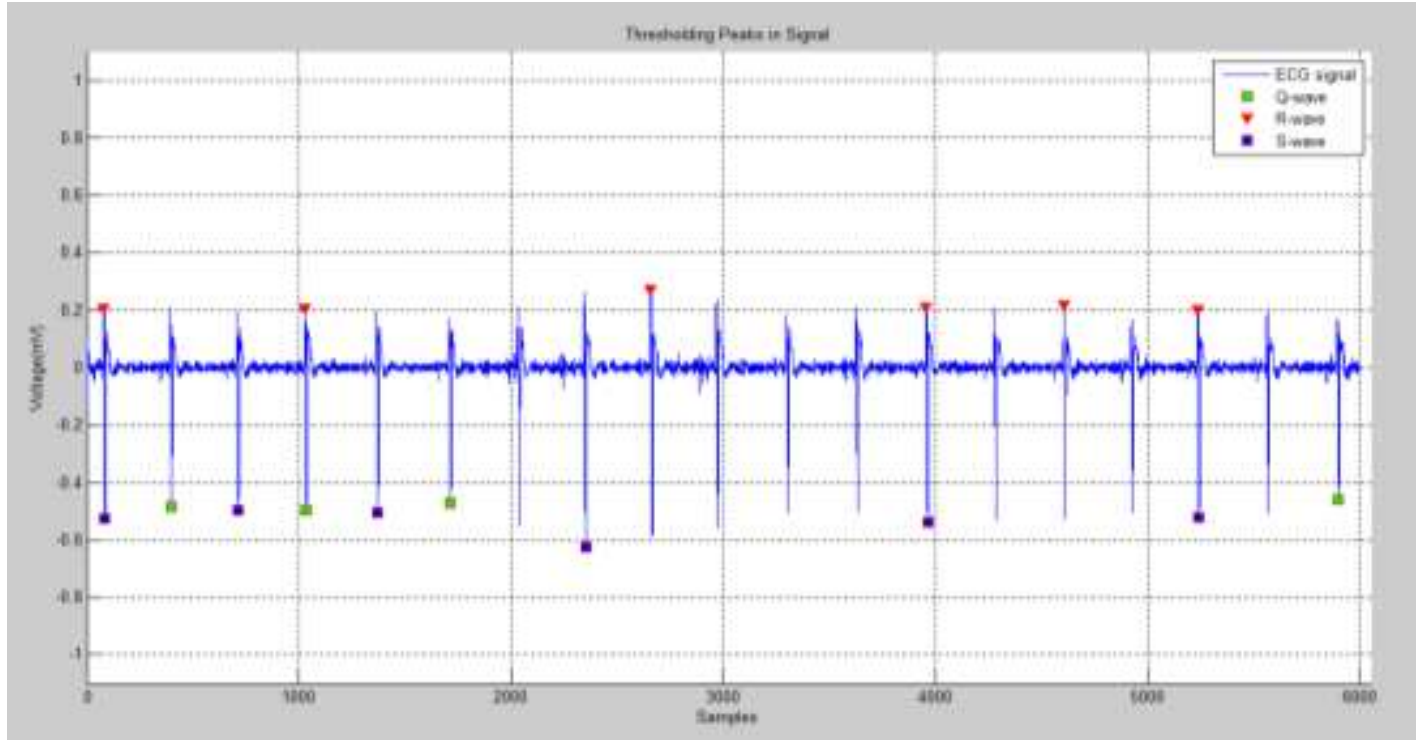

Figure 4: Detection of QRS Peak in ECG signal

\subsection{Hybrid Feature Extraction}

The Feature extraction stage is the key to the success for the ECG signal classification. The feature extraction discriminate the different types of signals in the given database. The features can be extracted from the ECG segmented contains arrhythmia or non-arrhythmias beat. Moreover, the feature extraction method provides the descriptive of the heartbeat segmentation. In this paper, Hybrid features are used to extract the features from heart beat segmentation using combination of DWT and DTCWT decomposition method.

\subsubsection{Discrete Wavelet Transform}

DWT provides good resolution at high frequency and good frequency resolution at low frequency. Due to this advantage, DWT can extract the local characteristics of the input heart beat segmentation. DWT decomposes the QRS complex using DWT techniques. The input signal is decomposed into low pass and high pass filter followed by down sampling in each stage. The ECG heart beat signal is decomposed into three level is illustrated in figure 5. In that Fig, the output of the first stage high pass filter provides the detail coefficient D1 and low pass filter provides the approximation coefficient A1. The Quadrature Mirror Filter (QMF) is used in each stage of low pass and high pass filter. QMF condition is satisfied using below equation:

$$
\begin{aligned}
& H(z) H\left(z^{-1}\right)+H(-z) H\left(-z^{-1}\right)=1 \\
& G(z)=z H\left(-z^{-1}\right)
\end{aligned}
$$

In the above equation $H(z)$ and $G(z)$ are the $z$ transform of low pass and high pass filter and it is related to time domain analyses is given by below form:

$$
g[L-1-n]=(-1)^{n} \cdot h[n]
$$

form:

Length of the filter is given by variable 'L'. The low pass and high pass filter for an output signal is given by below

$$
\begin{aligned}
& \operatorname{LPF}(k)=\sum_{n} x[n] \cdot h[-n+2 k] \\
& H P F(k)=\sum_{n} x[n] \cdot g[-n+2 k]
\end{aligned}
$$




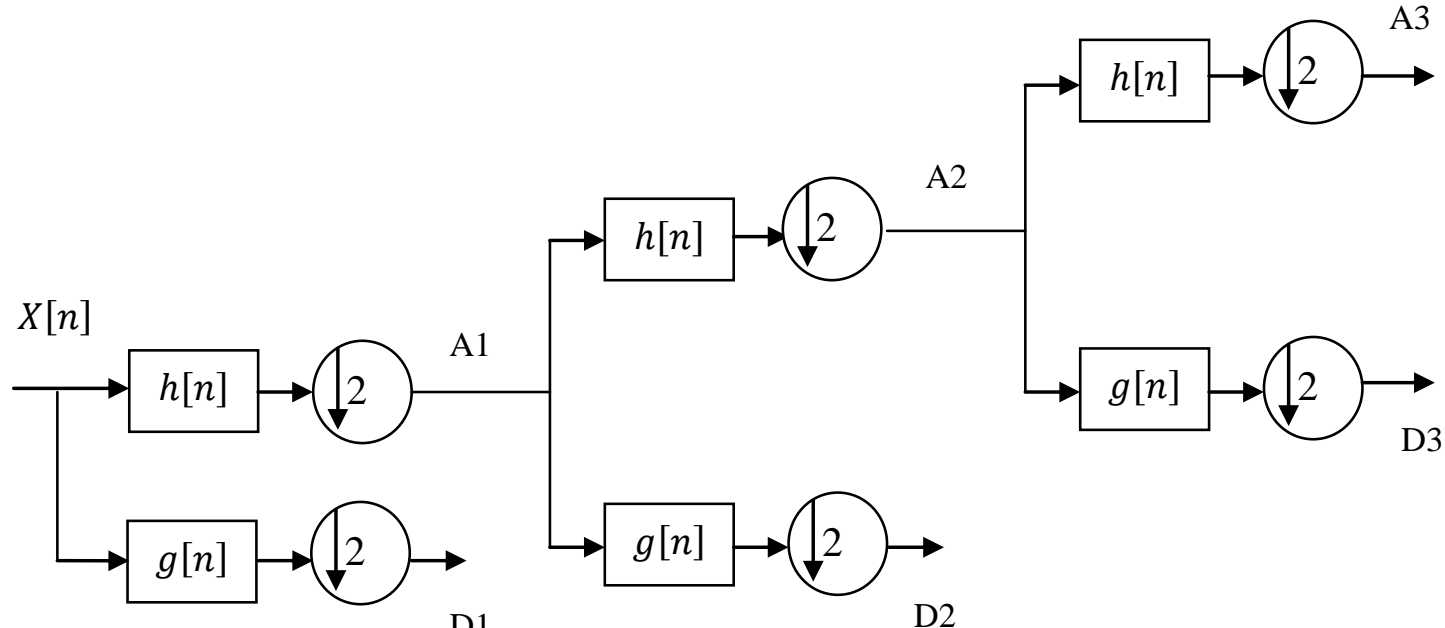

D1

D2

Figure 5: Three-level sub-band decomposition using DWT technique

\subsubsection{Dual Tree Complex Wavelet Transform}

The conventional DWT technique is a powerful tool for analyzing the ECG signal in a 1D signal but it has a drawback of oscillation, shift invariance and aliasing. It is very complicated to detect and analyse the signal with singularities because of wavelet coefficient oscillates around the singularities. The shift variance is occurred due to the down sampling operation at each stage. Hence, there is a change in the energy of the wavelet coefficient in the input pattern for a small time shift. This property leads to fail to distinguish the input signal shifts. Undecimated form of dyadic tree provides shift invariance but it suffers from increased computation requirements and high redundancy in the output. Aliasing is appear in the conventional DWT due to the calculation of wavelet coefficient using iterative time discrete operations with the non-ideal high and low pass filters. This aliasing property s canceled using inverse DWT, but only if the wavelet coefficients are not processed (Kadambe \& Srinivasan, 2006) [16].

To alleviate the problem of conventional method of DWT, a simple technique DTCWT is introduced. The magnitude of Fourier Transform does not oscillate positive and negative instead of these produce a small positive envelope on frequency domain. Second the shift invariant is perfect with the simple linear phase offset encoding with shift. Third, there is no aliasing part as well as no need of complicated aliasing cancelation property to reconstruct the signal. The below equation defines the Fourier transform which is based on complex valued oscillating sinusoids.

$$
e^{j w t}=\cos w t+j \sin w t
$$

The cosine and sine part in the above equation is derived from Hilbert transform pair and both parts are $90^{0}$ out of phase with each other. Based on Fourier transform inspiration, the DTCWT also employs complex valued wavelet and scaling function and it is given in below equation:

$$
\begin{gathered}
\varphi_{c}(t)=\varphi_{r}(t)+j \varphi_{i}(t) \\
\emptyset_{c}(t)=\emptyset_{r}(t)+j \emptyset_{i}(t)
\end{gathered}
$$

In the above two equation real part and the imaginary part of the complex wavelet function is represented by $\varphi_{r}(t)$ and $\varphi_{i}$ and they are Hilbert transform pair to each other. Two real wavelet filters are used in dual tree approach for acquired the real and imaginary part of the transform. Two filters are combined and defined as analytic filter. This filter gives a new structure and is similar to two standard DWT filter bank structure operating in parallel and is illustrated in figure.

The fig shows two part are called as Tree A and Tree B; where tree A is called as sub-band signal of upper DWT it defines the real part of the complex wavelet transform while Tree B is called as sub-band signal of lower DWT and it provides the imaginary part of the complex wavelet transform. Both Tree A and Tree B used different set of filters for the reconstruction.

Let low pass and high pas filter of the upper filter bank is represented as $h_{0}(n)$ and $h_{1}(n)$ in the same way low and high pass filter for the lower filter bank is denoted as $g_{0}(n)$ and $g_{1}(n)$. The low pass filter from both filter banks is designed such that wavelet functions are approximate Hilbert transform pair and they are related as

$$
g_{0}(n)=h_{0}(n-0.5)
$$


The below figure illustrates the structure of ID Q-shift dual tree proposed by (Kingsbury) where are two sets of filter was used; one set of filters for level and the other set of filters for higher level. There is even length and group delay of $1 / 4$ after the level 1 filter. The dual tree complex wavelet transform consists of two real DWT and the complex coefficients are occurred only if the two trees are combined.

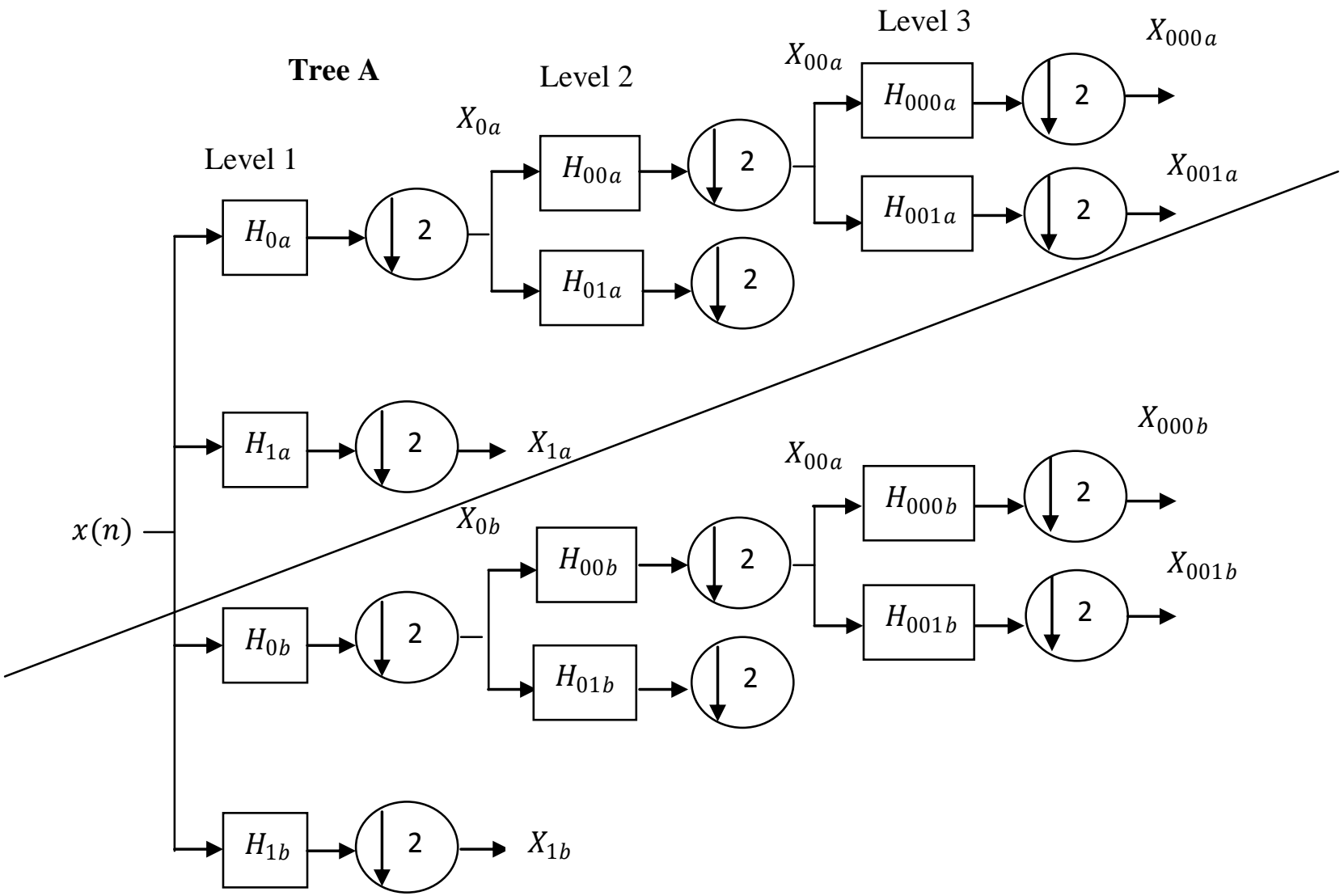

Tree B

Figure 6: Dual Tree Complex Wavelet Transform

Hybrid feature set are obtained using combination of DWT and DTCWT and is implemented as follows:

Hybrid features $=[$ DWT; DTCWT $]$

The DWT feature provides the details low frequency and high frequency of given QRS complex whereas DTCWT feature gives the shift invariance property of the QRS complex.

\subsection{Classification}

\section{The k-nearest neighbor ( $k N N)$}

The kNN method is one of the non parametric methods introduced by (Malini suvarna and Venkategowda, 2011) [17]. $\mathrm{kNN}$ is easy to implement and needs to be determine the number of nearest neighbor. Performance of kNN classification is based on number of nearest neighbor. kNN classification classifies the new samples from testing samples to the training samples depend on minimum distance function obtained from Euclidean distance.

The Euclidean distance between two points such as $\mathrm{X}_{1}=\left(\mathrm{x}_{11}, \mathrm{x}_{12}, \ldots, \mathrm{x}_{1 \mathrm{n}}\right)$ and $\mathrm{X}_{2}=\left(\mathrm{x}_{21}, \mathrm{x}_{22}, \ldots, \mathrm{x}_{2 \mathrm{n}}\right)$ can be obtained from below equation:

$$
\text { Euc. } \operatorname{dist}\left(\mathrm{X}_{1}, \mathrm{X}_{2}\right)=\sqrt{\sum_{\mathrm{i}=1}^{\mathrm{n}}\left(\mathrm{x}_{1 \mathrm{i}}-\mathrm{x}_{2 \mathrm{i}}\right)^{2}}
$$

The basic steps for k-NN algorithm are:

- Initialize the $\mathrm{k}$ value based on class.

- $\quad$ Calculate the Euclidean distance between the training and testing samples.

- $\quad$ Sort the obtained distance result in ascending order and take the minimum distance value as a predicted result. 


\section{Extreme Learning Machine}

In some existing approach, if the number of classes is increases the complexity of the overall classifier also increases [18]. The multicategory classification problem is reduced by the introduction of Neural Network (Linder, 2004) [19]. It can map the input data into different classes directly with one network. Basically, the parameters such as weights and biases of the feed forward ANN is tuned by the learning algorithm in their training scheme. Most of the gradient descent based learning algorithm in feed forward ANN are slower due to their improper learning steps and may converge to local minima. (Huang et al., 2004a, 2004b and 2005) [20, 21 and 22] proposed a new learning algorithm for Singlehidden Layer Feed Forward neural Network (SLFN) is Extreme Learning Machine.

The output of SLFN is represented as follows:

$$
f_{\widetilde{N}}(x)=\sum_{i=1}^{\tilde{N}} \beta_{i} G\left(a_{i}, b_{i}, x\right), x \in R^{n}, a_{i} \in R^{n}
$$

Where $\widetilde{N}$ defines the number of hidden layers, learning parameter of the hidden nodes are represented by $a_{i}$ and $b_{i}$ and $\beta_{i}$ represents the weight connecting the ith hidden node to the output node. $G\left(a_{i}, b_{i}, x\right)$ indicates the output of the ith hidden node with respect to the input $x$.

For the additive hidden node with activation function is represented as:

$$
G\left(a_{i}, b_{i}, x\right)=g\left(a_{i} . X+b_{i}\right), b_{i} \in R
$$

Where $a_{i}=\left[a_{i 1}, a_{i 2}, \ldots, a_{i n}\right]^{T}$ represents the weight vector connecting the ith hidden node and the input nodes, $b_{i}$ is the bias of the ith hidden node and $a_{i} . X$ represents the inner product of the $a_{i}$ and $X$ in $R^{n}$.

The two above equation can be written as:

$$
\begin{gathered}
H \beta=T \\
\text { Where } \\
H\left(a_{1}, \ldots, a_{N}\right)\left(b_{1}, \ldots, b_{N}\right)\left(x_{i}, \ldots, x_{n}\right)=\left[\begin{array}{ccc}
g\left(a_{1} \cdot x_{1}+b_{1}\right) & \ldots & g\left(w_{N} \cdot x_{1}+b_{N}\right) \\
\vdots & \ddots & \vdots \\
g\left(a_{1} \cdot x_{N}+b_{1}\right) & \ldots & g\left(a_{N} \cdot x_{N}+b_{N}\right)
\end{array}\right]_{N \times \widetilde{N}} \\
\beta=\left[\begin{array}{c}
\beta_{1}^{T} \\
\vdots \\
\beta_{N}^{T}
\end{array}\right]_{N \times m} \quad \text { and } T=\left[\begin{array}{c}
t_{1}^{T} \\
\vdots \\
t_{N}^{T}
\end{array}\right]_{N \times m}
\end{gathered}
$$

Where $\mathrm{H}$ represents the hidden layer output matrix of the network; the ith column and jth row of the hidden node output with respect to their input vector $x_{1}, x_{2}, \ldots, x_{N}$.

In real application the hidden nodes will be less than their training samples then only training error cannot be made exactly zero but can approach a non training error $\epsilon$. The hidden node parameter of SLFN need not be tuned in the time of training condition instead it simply be assigned with random values based on continuous sampling distribution. Equation (14) can be written as:

$$
\hat{\beta}=H^{\dagger} T
$$

Where $H^{\dagger}$ represents the Moore-Penrose generalized inverse of the hidden layer output matrix $H$. The ELM algorithm consist of three steps can be given as follows:

ELM Algorithm: Given a training set

$\mathrm{N}=\left\{\left(\mathrm{X}_{\mathrm{i}}, \mathrm{t}_{\mathrm{i}}\right) \mid \mathrm{X}_{\mathrm{i}} \in \mathrm{R}^{\mathrm{n}}, \mathrm{t}_{\mathrm{i}} \in \mathrm{R}^{\mathrm{m}}, \mathrm{i}=1, \ldots, \mathrm{N}\right\}$, activation function $\mathrm{g}(\mathrm{x})$, and hidden node number $\widetilde{\mathrm{N}}$.

STEP 1: Assign random hidden nodes by randomly generating parameters $\left(a_{i}, b_{i}\right)$ according to any continuous sampling distribution, $\mathrm{i}=1, \ldots, \widetilde{\mathrm{N}}$.

STEP 2: Calculate the hidden layer output matrix $\mathrm{H}$.

STEP 3: Calculate the output weight $\beta$

\section{RESULTS AND DISCUSSION}

$$
\beta=\mathrm{H}^{+} \mathrm{T}
$$

The Experimental results are carried out in MATLAB software package. The ECG signal is collected from the MIT-BIH database it consists of 48 recordings with duration of 30 minutes [23]. The sampling frequency of the respective database is $360 \mathrm{~Hz}$ and the resolution of the signal is 200 samples per mv. The database is divided into two classes are normal and abnormal class. To evaluate the performance of the proposed system using performance metrics such as sensitivity (Se), specificity (Sp) and Accuracy (ACC) which are given as follows:

Sensitivity defines the ratio of number of correctly detected events to the total number of events in the given events are calculated by below equation:

$$
S e=\frac{T P}{T P+F N} \times 100
$$

Where True Positive (TP) defines the total number of events and False Negative (FN) indicates the number of missed events

Specificity defines the ratio of the number of correctly rejected non-events to the total number of non-events is calculated using below equation:

$$
S p=\frac{T N}{T N+F P} \times 100
$$

Where True Negative (TN) defines the total number of non events and False Positive (FP) relates the number of falsely detected events.

The overall accuracy for the respective event is given below: 


$$
A c c=\frac{T P+T N}{T P+F N+T N+F P} \times 100
$$

Table 1: Performance comparison of the different events using ELM methods

\begin{tabular}{|c|c|c|c|c|c|c|}
\hline \multirow{2}{*}{ Events } & \multicolumn{3}{|c|}{ DWT-ELM } & \multicolumn{3}{c|}{ Hybrid features with ELM } \\
\cline { 2 - 7 } & Se & Sp & Acc & Se & Sp & Acc \\
\hline LBBB & 0.82561 & 0.627639 & 69.34 & 0.866667 & 0.648649 & 71.15 \\
\hline RBBB & 0.795319 & 0.589534 & 64.90 & 0.823529 & 0.619565 & 67.46 \\
\hline Normal & 0.781920 & 0.69782 & 73.88 & 0.818182 & 0.71875 & 75.92 \\
\hline
\end{tabular}

Table 2: Performance comparison of the different events using kNN methods

\begin{tabular}{|c|c|c|c|c|c|c|}
\hline \multirow{2}{*}{ Events } & \multicolumn{3}{|c|}{ DWT-ELM } & \multicolumn{3}{c|}{ Hybrid features with kNN } \\
\cline { 2 - 7 } & Se & Sp & Acc & Se & Sp & Acc \\
\hline LBBB & 0.942 & 0.97 & 96.24 & 0.964286 & 1 & 98.14815 \\
\hline RBBB & 0.89 & 0.98 & 92.34 & 0.9 & 1 & 94.44444 \\
\hline Normal & 0.94 & 0.98 & 96.45 & 0.961538 & 1 & 98 \\
\hline
\end{tabular}

Table $1 \& 2$ provides the comparison of different events using performance metrics such as sensitivity, specificity and accuracy using two different classifier ELM and kNN. The performance metrics are evaluated for two different types of diseases or events are LBBB, RBBB and also comparison are carried over for normal ECG signal. The metrics are obtained on the ECG signal using kNN and ELM classifier with the hybrid features generated by the combination of (DWT-DTCWT) and DWT features. The ECG signal were divided into two separate datasets are training and testing dataset.

The training dataset was used to train the kNN \& ELM classifier using three different types of heart beats whereas the testing dataset was used to testing the three classes of ECG signal and it verify the effectiveness of kNN and ELM classifier.

It is obvious from the table $1 \& 2$ that the higher accuracy is given by RBBB \& LBBB using kNN with hybrid feature compared to kNN with DWT features, ELM with hybrid feature and ELM with DWT feature. From this analysis observed that hybrid features overcomes that DWT features.

Also observed that hybrid feature with kNN classifier provides better accuracy compared to hybrid feature with ELM classifier because proposed hybrid feature have an advantage of both individual features as well as kNN classifier is good for small dataset with small set of features. The ELM classifier also used as regression and classification with given set of feature mapping function and it have ability to handle both large and small dataset it have a drawback of unnecessary input weight and hidden layer basis due to random selection of input weight and hidden layer.

\section{CONCLUSION}

In this paper, we proposed an ECG beat classification based on hybrid features and classifier. The hybrid features are extracted from QRS complex of the ECG signal. In this paper supervised learning approach of kNN and ELM classifier is used as a classifier because it provides good results compared to unsupervised classifier and it is easy to implement. The proposed method of hybrid features with kNN classifier provides an promising sensitivity, specificity and accuracy compared to hybrid feature with ELM classifier. The results reveals that kNN classifier is an good method for computer aided diagnosis of cardiac arrhythmias. The Experimental results also reveal that the performance of the hybrid feature with kNN classifier is better than DWT feature with kNN and ELM classifier.

\section{REFERENCES}

(1) Yuksel Ozbay and Gulay Tezel, "A new method for classification of ECG arrhythmias using neural network with adaptive activation function”, Digital Signal Processing, 1040-1049, 20 (2010).

(2) M.G. Tsipouras, D.I. Fotiadis, Automatic arrhythmia detection based on time and time-frequency analysis of heart rate variability, Computer Methods and Programs in Biomedicine 74 (2004) 95-108.

(3) Eduardo José da S. Luz, William Robson Schwartz, Guillermo Cámara-Cháveza, David Menotti, "ECG-based heartbeat classification for arrhythmia detection: A survey", Computer Methods and Program in Biomedicine, 2015.

(4) M.G. Tsipouras, D.I. Fotiadis, D. Sideris, An arrhythmia classification system RR-interval signal, Artificial Intelligence in Medicine 33 (2005) 237-250.

(5) Berat Dogan and Mehmet Korürek, "A new ECG beat clustering method based on kernelized fuzzy c-means and hybrid ant colony optimization for continuous domains", Applied Soft Computing 12 (2012) 3442-3451.

(6) Yakup Kutlu and Damla Kuntalp, "A multi-stage automatic arrhythmia recognition and classification system", Computers in Biology and Medicine 41 (2011) 37-45. 
(7) A. Koski, "Modelling ECG signals with Hidden Markov models", Artificial Intelligence in Medicine 8 (1996) 453471.

(8) Elif Derya Übeyli, "Adaptive neuro-fuzzy inference system for classification of ECG signals using Lyapunov exponents", computer methods and programs in biomedicine 93 (2009) 313-321.

(9) Ataollah Ebrahim Zadeh, Ali Khazaee and Vahid Ranaee, "Classification of the electrocardiogram signals using supervised classifiers and efficient features", computer methods and programs in biomedicine 99 (2010) 179194.

(10) M.R. Homaeinezhad , S.A. Atyabi, E. Tavakkoli, H.N. Toosi, A. Ghaffari and R. Ebrahimpour, "ECG arrhythmia recognition via a neuro-SVM-KNN hybrid classifier with virtual QRS image-based geometrical features", Expert Systems with Applications 39 (2012) 2047-2058.

(11) Mohit Kumar, Ram Bilas Pachori and U. Rajendra Acharya , "Characterization of coronary artery disease using flexible analytic wavelet transform applied on ECG signals", Biomedical Signal Processing and Control 31 (2017) 301-308.

(12) Roshan Joy Martis, U. Rajendra Acharyaa and Lim Choo Mina, "ECG beat classification using PCA, LDA, ICA and Discrete Wavelet Transform", Biomedical Signal Processing and Control 8 (2013) 437-448.

(13) S.S. Mehta and N.S. Lingayat, "Development of SVM based classification techniques for the delineation of wave components in 12-lead electrocardiogram", Biomedical Signal Processing and Control 3 (2008) 341-349.

(14) S.N. Yu, Y.H. Chen, Electrocardiogram beat classification based on wavelet transformation and probabilistic neural network, Pattern Recognit. Lett. 28 (2007) 1142-1150.

(15) A. Khazaee, A. Ebrahimzadeh, Classification of electrocardiogram signals with support vector machines and genetic algorithms using power spectral features, Biomed. Signal Process. Control 5 (2010) 252-263.

(16) Kadambe S, Srinivasan P. Adaptive wavelets for signal classification and compression. Int J Electron Commun (AEÜ) 2006; 60:45-55.

(17) Malini suvarna. and Venkategowda N., "Performance Measure and Efficiency of Chemical Skin Burn Classification Using KNN Method", Procedia Computer Science, 70, 48 - 54, 2015.

(18) N. Ghoggali, F. Melgani, Y. Bazi, A multiobjective genetic SVM approach for classification problems with limited training samples, IEEE Transactions on Geoscience and Remote Sensing 47 (2009) 1707-1718.

(19) R. Linder, D. Dew, H. Sudhoff, D. Theegarten, K. Remberger, S.J. Poppl, and M. Wagner, "The 'Subsequent Artificial Neural Network' (SANN) Approach Might Bring More Classificatory Power to ANN-Based DNA Microarray Analyses," Bioinformatics, vol. 20, no. 18, pp. 3544-3552, 2004.

(20) G.-B. Huang, Q.-Y. Zhu, and C.-K. Siew, "Extreme Learning Machine: A New Learning Scheme of Feed forward Neural Networks," Proc. Int'l Joint Conf. Neural Networks (IJCNN '04), July 2004.

(21) G.-B. Huang and C.-K. Siew, "Extreme Learning Machine: RBF Network Case," Proc. Eighth Int'I Conf. Control, Automation, Robotics, and Vision (ICARCV '04), Dec. 2004.

(22) G.-B. Huang and C.-K. Siew, "Extreme Learning Machine with Randomly Assigned RBF Kernels," Int'l J. Information Technology, vol. 11, no. 1, 2005.

(23) MIT BIH arrhythmia database- https://www.physionet.org/physiobank/database/mitdb/ 